\title{
In-vivo precision of a non-invasive ultrasound- based device to measure pelvic tilt for THA
}

\author{
Guillaume Dardenne ${ }^{1,2}$, Jean-Philippe Pluchon ${ }^{1,2}$, Hoel Letissier ${ }^{1,2,3}$, Aziliz \\ Guezou-Philippe ${ }^{1,2,3}$, Romain Gérard ${ }^{4}$, Christian Lefèvre ${ }^{1,2,3}$, and Eric \\ Stindel ${ }^{1,2,3}$ \\ ${ }^{1}$ LaTIM, Inserm, UMR 1101, SFR IBSAM, Brest, France \\ ${ }^{2}$ CHU Brest, Brest, France \\ ${ }^{3}$ Université de Bretagne Occidentale, Brest, France \\ ${ }^{4}$ Clinique Keraudren - Grand Large, Service d'Orthopédie, Brest, France \\ guillaume.dardenne@univ-brest.fr
}

\begin{abstract}
The cup orientation plays a major role in the long-term implant stability following Total Hip Arthroplasty (THA). Because of the patient specific spine-hip kinematics, the safe zone introduced by Lewinnek is more and more controversial. Several solutions have been recently developed to take into account such parameter for THA but are all either invasive, difficult to use or expensive. A non-invasive ultrasound (US) based device has been recently proposed which allows the acquisition of the pelvic tilt in different daily positions. The goal of this study is to analyze the in-vivo intra and inter-observer precision of this device. Measurements were realized by three physicians on three healthy subjects having a low, medium and high Body Mass Index (BMI). Among the three physicians, there were an expert, an intermediate, and a novice user. For each subject, the pelvic tilt was measured ten times by the three physicians in the supine, standing and sitting positions. The inter and intra-observer precisions have been analyzed using the intraclass correlation coefficient (ICC) and according to the BMI, the positions and the user expertise level. The inter-observer precision was therefore excellent whatever the BMI. It was also excellent regarding the supine and the sitting positions and good concerning the standing position. The in-vivo intra-observer precision was excellent for all measurements and whatever the user's expertise, the BMI and the positions. This study shows therefore that the precision of our system meets the clinical requirement. Introduction
\end{abstract}




\section{Introduction}

For several decades, Total Hip Arthroplasty (THA) has become the treatment of choice for advanced hip osteoarthritis. However, this intervention is still prone to complications. Dislocation is the second most common complication after aseptic loosening and is very influenced by the acetabular cup orientation. This last decade, more and more authors mentioned that the safe zone proposed by Lewinnek (Lewinnek, Lewis, Tarr, Compere, \& Zimmerman, 1978) is not suitable for every patients because of the patient specific spine-hip kinematics (Rivière et al., 2017).

Several solutions have been developed in order to take into account this parameter for THA (Kochman et al., 2017; Lazennec et al., 2015; Pierrepont et al., 2016). All of them are either invasive, difficult to use or expensive. A non-invasive ultrasound (US) based device has been recently proposed which allows the acquisition of the pelvic tilt, i.e. the angle between the Anterior Pelvic Plane (APP) and the vertical, in several daily positions: in the supine, standing and sitting positions (Dardenne et al., 2018). The accuracy of this system has been analyzed according to a mechanical test bench. This accuracy was very satisfying with an error of $1.15 \pm 0.82^{\circ}$ whatever the tilt and the soft tissue thickness.

The goal of this paper is therefore to in-vivo evaluate the intra and inter-observer precision of this device.

\section{Material and Methods}

The device is composed of a two-dimensional US probe (L12-5L60N, Telemed $\left.{ }^{\circledR}\right)$ connected to a $^{\circ}$ tab (Surface Pro 3, Microsoft ${ }^{\circledR}$ ) which is equipped with an accelerometer and a front-facing RGB camera (figure 1). The three-dimensional (3D) position and orientation of the US probe is determined thanks to an ARUCO marker rigidly fixed to the US probe and localized by the front-facing camera. Both the US probe and the RGB camera have been beforehand calibrated. The APP is determined by acquiring with the US probe the right anterior iliac spines, the left anterior iliac spines and the pubic symphysis. Landmarks are then manually placed by the user on the three US images. The pelvic tilt is finally estimated by computing the angle between the APP, defined by the three landmarks, and the vertical provided by the embedded tab accelerometer. 


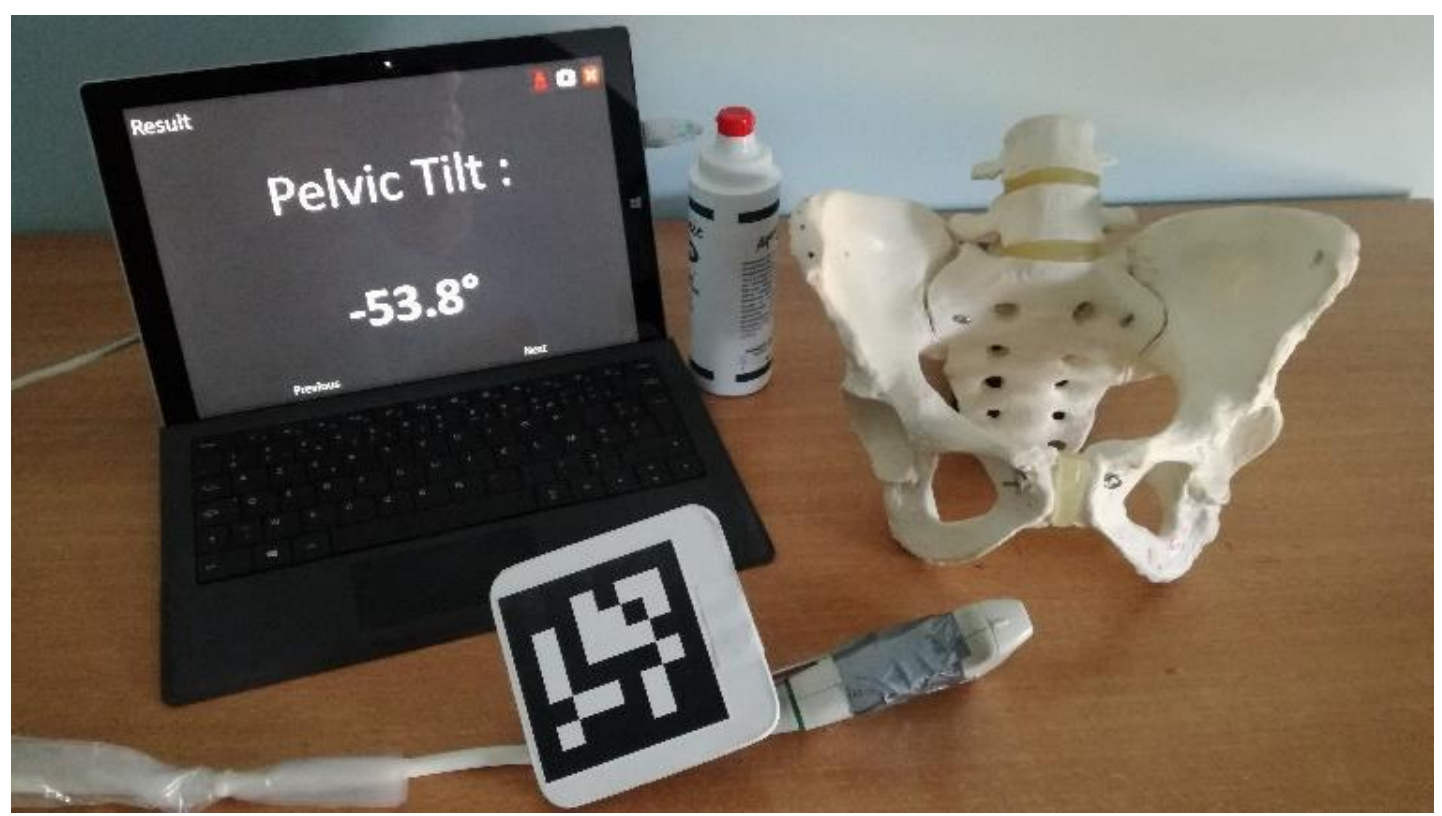

Figure 1: Device including the tab and the US probe equipped with an ARUCO marker

To evaluate the precision, pelvic tilt measurements were realized with the device on three healthy subjects and by three physicians. These subjects had a low, medium and high Body Mass Index (BMI), i.e. respectively with a BMI less than 20, a BMI between 24 and 26, and a BMI greater than 30 . Among the three physicians, there were an expert who used the device on ten patients before the study, an intermediate who never used the device before but is experienced in osteoarticular US, and a novice who never used the device before and is not experienced in US. For each subject, the pelvic tilt was measured ten times by the three physicians in the supine, standing and sitting positions, resulting in 90 measurements per subject. The inter and intra-observer precisions have been analyzed according to the $\mathrm{BMI}$ and the positions using the intraclass correlation coefficient (ICC). The criteria defined by Cohen were used: low precision (ICC $<0.4)$, medium precision $(0.40<$ ICC $<0.59)$, good precision $(0.60<$ ICC $<0.74)$ and excellent precision $(0.75<$ ICC $<1.0)$.

\section{Results}

For the inter observer precision, the ICC was $0.998,0.9981$ and 0.996 for respectively a low, moderate and high BMI. The ICC was $0.983,0.925$ and 0.69 for respectively the supine, sitting and standing positions.

For the intra observer precision, the ICC was 0.999 for low and medium BMI, whatever the user's expertise. For high BMI, the ICC was 0.992, 0.998 and 0.996 for respectively the expert, intermediate and novice users. Regarding the positions, the ICC was $0.984,0.981$ and 0.977 for the supine position, $0.874,0.942$ and 0.787 for the standing position, and $0.868,0.955$ and 0.986 for the sitting position, for respectively expert, intermediate and novice users. 


\section{Discussions}

The in-vivo inter-observer precision was therefore excellent for all measurements and excellent according to the BMI. The inter-observer precision was also excellent regarding the supine and the sitting positions and good concerning the standing position. The intra-observer precision was excellent for all measurements, whatever the user's expertise, the BMI and the positions.

This study shows therefore that besides the accuracy, which has been studied last year (Dardenne et al., 2018), the inter and intra-observer precision of our system meets the clinical requirement.

\section{References}

Dardenne, G., Pluchon, J.-P., Guezou-Philippe, A., Letissier, H., Hamitouche, C., Lefèvre, C., \& Stindel, E. (2018). An Easy-to-Use Portable Ultrasound Based Device for the Measurement of the Pelvic Tilt. In EPiC Series in Health Sciences (Vol. 2, pp. 39-34). EasyChair. https://doi.org/10.29007/pwhr

Kochman, A., Goral, A., Martin, T., Marek, W., Kozak, J., Morawska-Kochman, M., \& Synder, M. (2017). Application of Navigated Ultrasound for Assessment of the Anterior Pelvic Plane in Patients With Degenerative Hip Diseases. Journal of Ultrasound in Medicine, 36(7), 1373-1380. https://doi.org/10.7863/ultra.16.07016

Lazennec, J.-Y., Rousseau, M.-A., Brusson, A., Folinais, D., Amel, M., Clarke, I., \& Pour, A. E. (2015). Total Hip Prostheses in Standing, Sitting and Squatting Positions: An Overview of Our 8 Years Practice Using the EOS Imaging Technology. The Open Orthopaedics Journal, 9, 26-44. https://doi.org/10.2174/1874325001509010026

Lewinnek, G. E., Lewis, J. L., Tarr, R., Compere, C. L., \& Zimmerman, J. R. (1978). Dislocations after total hip-replacement arthroplasties. The Journal of Bone and Joint Surgery. American Volume, 60(2), 217-220. Retrieved from http://www.ncbi.nlm.nih.gov/pubmed/641088

Pierrepont, J. W., Stambouzou, C. Z., Miles, B. P., O’Connor, P. B., Walter, L., Ellis, A., ... Marel, E. (2016). Patient-specific Component Alignment in Total Hip Arthroplasty. Reconstructive Review, 6(4). Retrieved from https://www.reconstructivereview.org/ojs/index.php/rr/article/view/148/202

Rivière, C., Lazennec, J.-Y., Van Der Straeten, C., Auvinet, E., Cobb, J., \& Muirhead-Allwood, S. (2017). The influence of spine-hip relations on total hip replacement: A systematic review. Orthopaedics \& Traumatology: Surgery \& Research, 103(4), 559-568. https://doi.org/10.1016/j.otsr.2017.02.014 